\title{
Infrared polarimetry: gaining a molecular view of polymer viscoelasticity
}

\author{
Julia Anne Kornfield
}

Julia Anne Kornfield, "Infrared polarimetry: gaining a molecular view of polymer viscoelasticity," Proc. SPIE 1636, Applied Spectroscopy in Materials Science II, (14 May 1992); doi: 10.1117/12.59291 


\title{
Infrared Polarimetry: Gaining a Molecular View of Polymer Viscoelasticity
}

\author{
Julia A. Kornfield \\ California Institute of Technology \\ Chemical Engineering \\ Pasadena, CA 91125
}

\begin{abstract}
Recent exciting advances have been made using infrared (IR) diode lasers to obtain sensitive and rapid measurements of molecular-level dynamics that control polymer material properties. Infrared diodes provide a bright, nearly monochromatic, polarized source that is excellent for use in polarimetry. Optical polarimetry is the measurement of the state of polarization of light. It is a powerful technique for studying molecular and microstructural anisotropy in a sample by measuring how the sample alters the polarization of a transmitted beam. In polymers, it is precisely such molecular anisotropy that underlies their viscoelastic properties. As theories are developed to predict the relaxation dynamics of individual parts of a polymer molecule, and of different species in polymer blends, there is increasing interest in experimental methods to distinguish these dynamics. Infrared polarimetry is extremely valuable in this regard, not only because of the direct relationship between the IR dichroism and molecular orientation, but also because it is amenable to labeling. The present paper reviews the basis of this technique and its application to study the effects of polydispersity on melt rheology, and the relaxation dynamics at different positions along a polymer chain. It concludes with an overview of research in progress and future problems to be tackled.
\end{abstract}

\section{INTRODUCTION}

Manufacture of articles from polymer materials invariably involves processing the polymer in a liquid state, either in solution or in the melt. Within the liquid the polymer molecules are long, flexible or somewhat stiff, linear or branched structures containing hundreds to thousands of backbone bonds. When these large molecules are distorted by flow, they give rise to stresses in the fluid that ultimately decay to zero only after the conformation of the molecules has fully equilibrated. The longest relaxation times of the chain are very sensitive to its overall length and topology. If a deformation is imposed rapidly, on a timescale much shorter than this terminal relaxation, the liquid appears elastic; only if the deformation is slow compared to the terminal relaxation time is the behavior similar to that of a purely viscous liquid. On intermediate time scales, the fluid shows intermediate "viscoelastic" character.

One of the great challenges in polymer physics is to explain why the relaxation dynamics have the particular time dependences that they do, and why they change with concentration, molecular weight, and molecular architecture the way they do. Molecular theories have been developed for linear chains in solutions, in low molecular weight melts, and in high molecular weight melts. ${ }^{1}$ The latter case has also been treated for star-branched ${ }^{2}$ and H-shaped molecules. ${ }^{3}$ In high molecular weight melts, the dynamics of any chain are strongly coupled to those of surrounding chains. So, the viscoelastic properties of the melt are non-linear functions of the molecular weight distribution (polydispersity). When chains of different types are dissolved in one another (miscible blends), the effect of differences in chain length and chemical structure must both be considered. ${ }^{4}$ Finally, 
polymers that consist of long chains of chemically different repeat units, covalently linked to each other (block copolymers) can have a wide range of disordered liquid and ordered liquid-crystal structures that influence the relaxation dynamics. ${ }^{5}$ These are just beginning to be treated theoretically.

While the most common test of molecular models of polymer rheology is their success in describing the macroscopic vicoelastic response of polymers, ${ }^{6}$ the ultimate test of their validity is their success in describing the underlying molecular relaxation dynamics. For example, in linear polymers, such theories not only predict the overall relaxation, but also the relaxation of any particular segment along the chain. ${ }^{7}$ Models of bidisperse blends predict the relaxation of the long chains and the short chains in the blend as functions of their respective lengths and concentrations. Similarly, models of miscible blends describe the dynamics of the individual components, and those of $\mathrm{H}$-shaped polymers predict the relaxation of the topologically distinct parts (arms or backbone) of the macromolecule. In order to obtain such information experimentally, optical methods are used together with labeling techniques, usually with an anisotropic fluorescent dye or with an isotopic label. The latter approach is preferable, because the substitution of a small amount of hydrogen by deuterium is usually sufficient as an infrared label; this perturbs the chemical structure much less than a bulky dye molecule.

Once a particular segment of a given polymer or a particular species in a mixture has been labeled, its relaxation dynamics can be monitored using infrared dichroism. Dichroism is the anisotropy in the attenuation of light by the sample. The infrared dichroism is proportional to the second moment of the orientation distribution of the molecular axis (transition moment vector) of the group responsible for vibrational absorption at the wavelength of interest. When deuterium is used as the label, a carbon-deuterium stretching absorption is particularly useful for monitoring the labeled group, because very few chemical structures absorb in the region $2195-2020 \mathrm{~cm}^{-1}$. To measure infrared dichroism in a polymer under flow, it is desireable to have a bright source of polarized light at the frequency of the labeled absorption. Using an IR diode laser source with polarization-modulation polarimetry techniques, one can rapidly measure dichroism even in very slightly deformed polymer melts - to which many theories are restricted.

The following sections describe the relationship between the optical observables and molecular orientation, the design of the apparatus used to measure them, and the results obtained regarding the effects of polydispersity and of chain topology on polymer dynamics.

\section{INFRARED DICHROISM AND BIREFRINGENCE}

\subsection{OPTICAL ANISOTROPY AND MOLECULAR ORIENTATION}

The starting point for molecular theories of viscoelasticity is the representation of the real chain by a coarse-grained model. If we consider a long enough section of the chain, it will behave statistically like a random flight or Gaussian chain. If its mean end-to-end distance is perturbed, it will exert a restoring force, and for small perturbations it will act like a Hookean spring. Relative motion of this section with respect to its neighbors will produce some drag force. To represent these two effects we model such a segment of chain as a bead and spring. A string consisting of $N$ of these segments is used to represent the polymer.

The length and orientation of the $n$-th segment is described by $\mathbf{r}_{n}$. The state of stress in the melt is related to segmental orientation by

$$
\sigma_{i j}=A<r_{i} r_{j}>+P \delta_{i j}
$$


where $\sigma_{i j}$ is the stress tensor, $\left\langle r_{i} r_{j}\right\rangle$ is the second moment of the orientation distribution of the segments, $P$ is the pressure, $\delta_{i j}$ is the identity tensor, and $A=\left(3 k T c / b^{2}\right)$ where $k T$ is the thermal energy, $c$ is the number concentration of segments, and $b^{2}$ is the mean-square length of the segments. Anisotropy in the real refractive index of a polymer under stress leads to a refractive index tensor that is also found to be the sum of a term proportional to the second moment tensor $<\mathbf{r r}>$ plus an isotropic term. Thus, for homopolymer melts we can obtain essentially the same information by measuring either the stress or the birefringence (anisotropy in the real refractive index).

To isolate the contribution to the stress of a particular part of a chain or of a particular species in a mixture, we seek to determine the second moment of the orientation of the segments in just that portion of the polymer or component of the melt. For that purpose, we introduce an isotopic label in precisely that portion or component, and observe the anisotropy in the attenuation of light at an infrared absorption unique to the label. This is described by the imaginary part of the refractive index tensor, $n_{i j}{ }^{\prime \prime}$, and it is proportional to the second moment of the local molecular orientation of just the labeled segments. Because the local orientation of a monomer within a coarse-grained segment depends not only on the orientation of that segment, but also on the average orientation of the surrounding segments, $n_{i j}{ }^{\prime \prime}$ is given by the sum of two terms: one proportional to the second moment of the orientation distribution of the labeled segments, and one proportional to the second moment of the orientation of all segments in the melt: ${ }^{9}$

$$
n_{i j}^{\prime \prime}=C(\lambda)\left[<r_{i} r_{j}-\frac{1}{3} r^{2} \delta_{i j}>_{K}+\frac{\epsilon}{1-\epsilon}<r_{i} r_{j}-\frac{1}{3} r^{2} \delta_{i j}>\right],
$$

where $C(\lambda)$ is a wavelength dependent factor, subscript $K$ denotes labeled segments, and $\epsilon$ is a coefficient that describes the strength of orientational coupling. Knowing the overall orientation from either stress or birefringence measurements, one can determine the segmental orientation distribution of the labeled portion of the melt from the infrared dichroism (anisotropy in the imaginary refractive index).

This simple picture applies for the case of homopolymer systems, where the label may be used to distinguish topologically different parts of a given polymer or of different molecular weight species in the melt. When considering a block copolymer or a blend, the analysis is modified to account for the different optical and mechanical properties of the chemically distinct structures in the melt.

\subsection{OPTICAL APPARATUS}

The experimental arrangement used to simultaneously measure infrared dichroism and birefringence is an extension of the optical train developed by Fuller and coworkers to measure transient linear birefringence and dichroism at visible wavelengths. ${ }^{10}$ A schematic diagram of the optical train is shown in Fig. $1 .^{11}$ The light source is an infrared diode laser with a frequency range of $2180-2195 \mathrm{~cm}^{-1}$ and a continuous power rating of greater than $10 \mathrm{~mW}$. The beam is collimated and passed through polarization modulating optics before impinging on the sample. Then the beam is passed through the polymer sample, either a polymer melt in a parallel plate flow cell (two different designs are shown in Fig. 2), or a polymer solid film in a stretching device. The transmitted light is analyzed using two detectors.

To measure the dichroism of the sample, one detector observes the modulation in the intensity of the transmitted beam directly (Detector 1 in Fig. 1); 


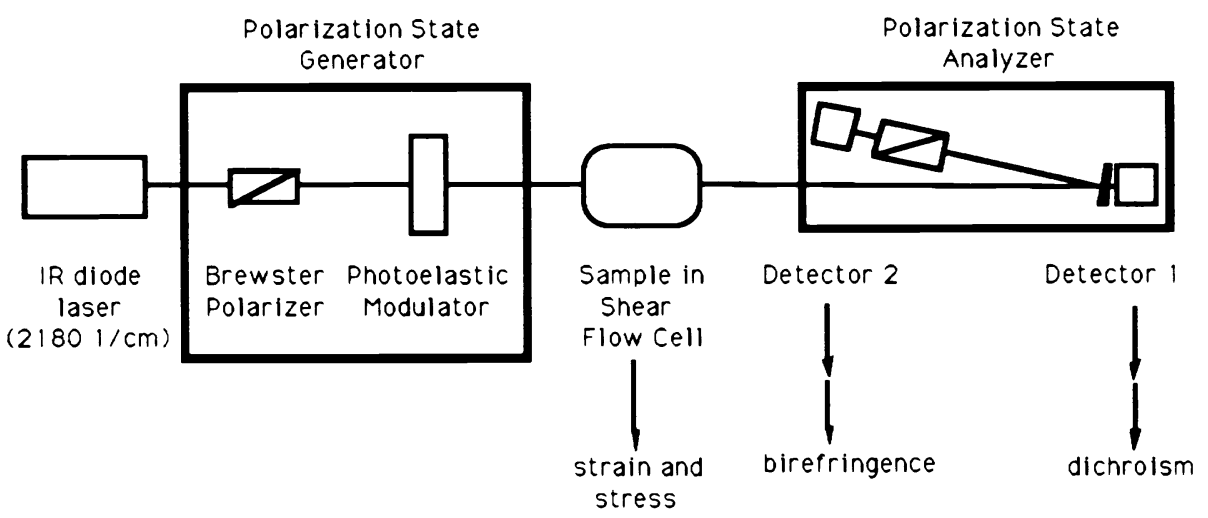

Figure 1. Schematic diagram of the optical train used for simultaneous measurement of IR dichroism and birefringence.

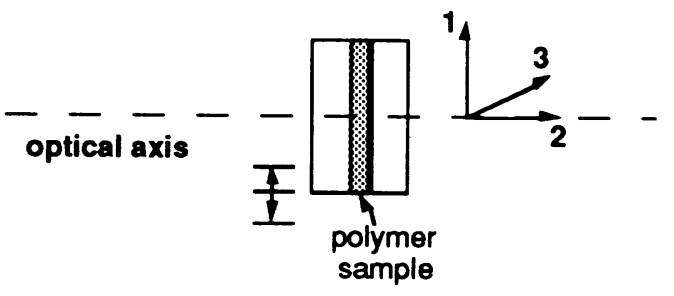

Figure 2. Schematic illustration of flow cell geometry. The flow direction is parallel to axis 1; the velocity gradient and the optical path are parallel to axis 2; and the vorticity axis is in direction 3 .

the signal is analyzed by a lock-in amplifier to determine the magnitude of the dichroism. To record the birefringence of the sample, part of the transmitted light is directed through analyzing optics that translate phase modulation into intensity modulation observed by Detector 2 in Fig. 1 ; this signal is demodulated to determine the magnitude of the birefringence. Both detectors are liquid nitrogen cooled, indium-antimonide, photovoltaic detectors.

In the first generation apparatus, the two optical signals are recorded with a measurement of the displacement of the flow cell. ${ }^{11}$ The polarization state generator (PSG in Fig. 1) consists of a Brewster angle polarizer and a zinc selenide photoelastic modulator; and the analyzing optics is simply a second Brewster angle polarizer. This apparatus is intended primarily for studying the time evolution of the optical signals, rather than their absolute magnitudes. A more recent appa- 
ratus based on the same principles records the two optical signals simultaneously with the stress and strain in the sample. ${ }^{12}$ To facilitate quantitative measurements of birefringence and dichroism, slightly different optics are used (a quarter wave plate is added both to the PSG and to the analyzing optics). ${ }^{10}$ The new apparatus can also study samples over a range of temperature from -70 to $400^{\circ} \mathrm{C}$ and of frequency from $16 \mathrm{~Hz}$ to $0.0016 \mathrm{~Hz}$, while the original apparatus is limited to 0 to $100^{\circ} \mathrm{C}$ and 4 to $0.006 \mathrm{~Hz}$.

\section{MOLECULAR ASPECTS OF POLYMER VISCOELASTICITY}

This relatively new experimental tool has already been applied to clarify a number of fundamental questions regarding the dynamics of homopolymers. What are the dynamics of relaxation of each species (long and short linear chains) in a bidisperse melt? How do they vary with the blend ratio of long and short polymer? Does the orientational coupling in a bidisperse homopolymer depend on the molecular weight of the chains? How so? In a nearly monodisperse homopolymer, how does the relaxation proceed at different positions along the length of the chain? The answers to these questions, along with prospects for further work, are given in the next two subsections.

Currently, research is underway to clarify dynamics in more complicated systems including liquid crystalline polymers, miscible blends, and block copolymers. These are described briefly in Section 3.3.

\subsection{EFFECTS OF POLYDISPERSITY}

Because the terminal relaxation time of a long polymer depends non-linearly on its length (roughly, $\tau \sim M^{3.4}$ ), the distribution of molecular weights in a polymer melt has a strong, non-linear effect on its viscoelastic properties. A bidisperse melt is a relatively simple model system to begin testing theoretical models of the effects of polydispersity. Imagine starting with a system of pure, very long chains, and gradually adding to it some much shorter, but still long chains. This introduces two, widely separated fundamentaltime scales for relaxation: the overall relaxation time of the long chains, and that of the short chains. Various models have been developed to describe how the dynamics of the long chains are altered by the addition of short chains to the melt; the models anticipate that the relaxation of the short chains will be only slightly slower in the bidisperse melt than it is in a monodisperse melt.

To discriminate among the models, infrared dichroism measurements were performed on a series of blends of the same fraction of long polymer $\Phi_{L}$, but with either some of the short chains or some of the long chains isotopically labeled. ${ }^{11}$ Simultaneous birefringence measurements were made to monitor the overall relaxation of the melt. Typical results are shown in Fig. 3 for a bidisperse poly (ethylenepropylene) (PEP) melt containing $30 \%$ long $(360 \mathrm{~kg} / \mathrm{mol})$ and $70 \%$ short (52 $\mathrm{kg} / \mathrm{mol}$ ) chains. Notice that the overall relaxation appears to have a fast mode of relaxation $\left(\tau_{S} \approx 0.4 s\right)$ and a slow mode $\left(\tau_{L} \approx 200 s\right)$. These time scales are close to those of the pure long and short components. Notice that the long chain dichroism relaxation also has qualitatively a bimodal relaxation time distribution with similar characteristic times. The short chain dichroism relaxes more rapidly than the bulk, but quite differently from its relaxation in a pure short chain melt, which has a terminal relaxation time of $0.4 \mathrm{~s}$. The measurable short chain dichroism beyond a time of a few seconds is due to the local orientational coupling between the short chains and the surroundings. When the effect of orientational coupling is included with $\epsilon=0.45$, the results for the bulk and each component in 


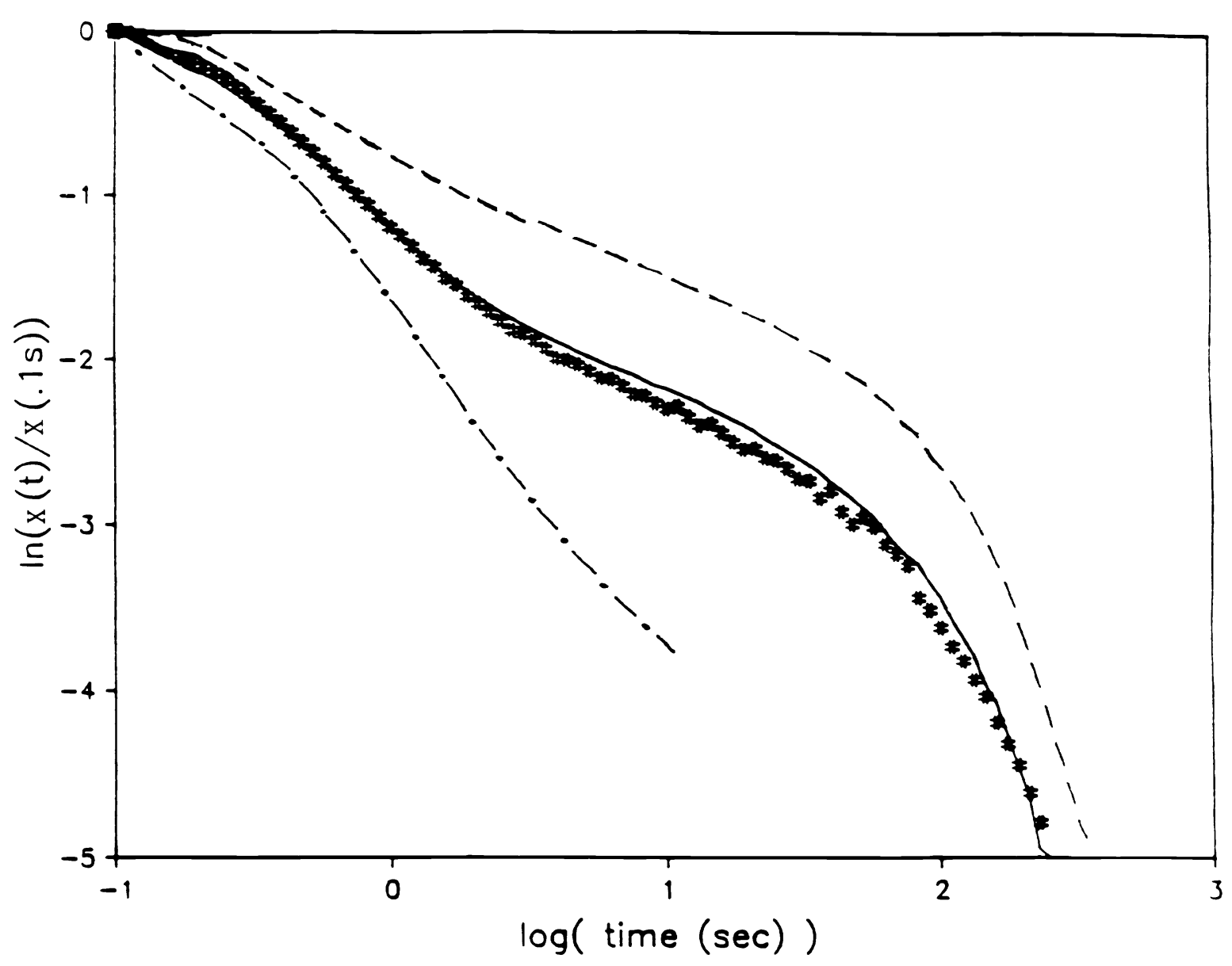

Figure 3. Step-strain relaxation of a bidisperse melt containing $30 \%$ vol. long polymer: long chain dichroism (dashed curve), the short chain dichroism (dashdot curve) and the bulk (solid curve for shear stress, \# symbols for birefringence). See ref. 11 for step strain and oscillatory shear response of blends of $0,10,20,30$, 50,75 and $100 \%$ long polymer.

melts with $\Phi_{L}=0,0.1,0.25,0.5,0.75,0.9$ and 1.0 , are all well described by the Rubinstein-Helfand-Pearson model. ${ }^{9}$

In order to investigate the possible molecular weight dependence of the orientational coupling that is evident in the short chain dichroism relaxation, subsequent experiments were performed with short chains of a range of molecular weights, holding the long chain length and volume fraction fixed. ${ }^{13}$ The results indeed show a dramatic effect of chain length: for polybutadiene chains of molar mass 0.5 to $2 \mathrm{~kg} / \mathrm{mol}$ a very strong coupling is observed with $\epsilon \approx 0.9$; the value drops to $\epsilon \approx 0.4$ as molecular weight is increased from 2 to $22 \mathrm{~kg} / \mathrm{mol}$. 
This molecular weight dependence is in contradiction to the decrease with decreasing molecular weight predicted by a configurational entropy model based on long chains of isotropic segments. ${ }^{14}$ Perhaps it will be explained when the theory is extended to the case of anisotropic segments.

\subsection{EFFECT OF POSITION ALONG A LINEAR CHAIN}

Both the Rouse model that describes low molecular weight polymer melts and the reptation model that describes high molecular weight melts predict the relaxation dynamics of each segment along the length of the chain. Qualitatively, both models predict that the relaxation is fastest for segments near the end of the chain and is progressively slower as you approach the center of the chain. Measurements of the segmental dynamics as a function of position can be used to test these models more stringently than is possible using overall stress or birefringence. ${ }^{15}$ Infrared polarimetry using diode lasers and polarization modulation has been used to obtain sufficiently accurate measurements of the relaxation spectrum of selectively labeled end- and center-segments that the results could be quantitatively compared to the Rouse and reptation models. ${ }^{7}$ The samples were well entangled (about thirty times the entanglement molecular weight, $M_{e}$ ); two different endlabeled samples were used, one with a $2.5 \times M_{e}$ end-block deuterated, and the other with $10 \times M_{e}$ deuterated. One center labeled sample was used, having a $2.5 \times M_{e}$ block deuterated. With independently determined values of the coupling coefficient $\epsilon$, Ylitalo et al. found good agreement with the reptation model not only for the overall relaxation of the chains, but for end- and center-blocks individually. The Rouse model captured some of the qualitative features, but did not agree quantitatively with the observed relaxation.

This approach was very successful. In the future it can be used to determine the nature of the cross-over behavior that occurs at intermediate molecular weights, with dynamics having some features of the Rouse model and some of the reptation model. Selectively labeled entangled polymers can also be used to determine in more detail the limitations of the reptation model in describing dynamics near the chain ends (due to fluctuations in contour length) and near the chain center (due to constraint release).

\subsection{FUTURE APPLICATIONS}

There is an enormous array of problems that should be studied using the enhanced speed and sensitivity of infrared diode laser based polarimetry. In the case of homopolymers, model predictions of the effects of chain branching on the relaxation of star and $\mathrm{H}$-polymers have not yet been tested quantitatively. Applied to miscible polymer blends, research is underway in Prof. Fuller's laboratory at Stanford, working on blends of poly(ethylene oxide) and poly(methyl methacrylate), and in our group, studying blends of 1,4-polyisoprene and 1,2-polybutadiene, and of polystyrene and poly(vinyl methyl ether). The results of these studies can be used to test and guide the extensions of molecular models to the case of miscible blends.

Another class of problems involves flow induced distortion not only of molecular orientation, but also of fluid microstructure. Examples include block copolymers where the pattern of concentration fluctuations in a disordered melt near the phase separation boundary, and the pattern of the microphase separated structure at lower temperatures, introduce a longer length- and time-scale to the problem. This will not only slow molecular relaxation, but add a new contribution to the stress altogether. Using a combination of optical methods, including infrared dichroism, we can determine the dynamics on these different levels. Analogous 
problems emerge in liquid crystalline polymers: in addition to molecular relaxation processes, the dynamics of larger scale orientational defects (disclinations) must be explored experimentally and understood theoretically. Macroscopic methods cannot help distinguish between these two types of underlying dynamics, but an appropriate choice of optical measurements can.

\section{CONCLUSION}

Infrared polarimetry is dramatically enhanced by the use of bright (up to $10 \mathrm{~mW}$ ), polarized diode laser sources. Applied to polymer science, this provides rapid, sensitive determination of the orientation of labeled chain segments, even when these are dilute and when the applied deformation is small. In contrast to Fourier Transform Infrared (FTIR) dichroism measurements using conventional "Glo-Bar" sources, the apparatus based on diode lasers can study:

- samples up to $2 \mathrm{~mm}$ thick so that both shearing of liquids and stretching of films can be used,

- slightly deformed samples, as small as $1 \%$ shear strain (in contrast to a typical value of $100 \%$ extension in FTIR dichroism experiments),

- both the orientation of the bulk and the labeled component simultaneously,

- transient response on timescales of $1 \mathrm{~ms}$ and longer.

This powerful experimental tool has already been applied to a number of problems in homopolymer melts: the effects of polydispersity, the nature of local orientational coupling in melts, and the relaxation of end- and center-blocks of a linear chain. Many additional problems remain to be investigated using this method to observe molecular-level dynamics in branched chains, polymer blends, block copolymers and liquid crystalline polymers. In each case the results can be used to test and to guide theories of polymer viscoelasticity, enhancing our ability to predict and control polymer material properties.

\section{REFERENCES AND NOTES}

1. For a summary of the Zimm model of polymer solutions, the Rouse model of low molecular weight melts, and the reptation model of high molecular weight melts, with references to the original works, see M. Doi and S. F. Edwards, The Theory of Polymer Dynamics, Clarendon Press, Oxford (1988).

2. M. Doi and N. Kuzuu, J. Polym. Sci., Polym. Lett., 18, 775 (1980); D. S. Pearson and E. Helfand, Macromolecules, 17, 888 (1984).

3. T. C. B. McLeish, Macromolecules, 21, (1988).

4. C. Tsenoglou, J. Polym. Sci., Polym. Phys. Ed., 26, 2329 (1988).

5. F. S. Bates, Science, 251, 898 (1991).

6. J. P. Montfort, G. Marin, D. Monge, Macromolecules, 17, 1551 (1984); H. Watanabe, T. Kotaka, Macromolecules, 17, 2316 (1984); H. Watanabe, T. Sakamoto, T. Kotaka, Macromolecules, 18, 1008, 1436 (1985); M. Struglinski, W. W. Graessley, Macromolecules, 18, 2630 (1985).

7. C. M. Ylitalo, G. G. Fuller, V. Abetz, R. Stadtler, D. S. Pearson Rheologica Acta, 29, 543 (1990).

8. For example, M Rubinstein, E. Helfand, D. S. Pearson, Macromolecules, 20, $822(1987)$. 
9. M. Doi, D. S. Pearson, J. A. Kornfield and G. G. Fuller, Macromolecules, 22, $1488(1989)$.

10. S. J. Johnson, P. L. Frattini, G. G. Fuller, Coll. Int. Sci., 104, 440 (1985).

11. J. A. Kornfield, G. G. Fuller and D. S. Pearson, Macromolecules, 22, 1334 (1989); J. A. Kornfield, G. G. Fuller and D. S. Pearson, Macromolecules, 24, 5429 (1991).

12. K. Rangaramanujam, J. Lai, J. Kornfield, manuscript in preparation.

13. C. M. Yilitalo, G. G. Fuller, J. Non-Crys. Solids, 131, 676 (1991).

14. E. A. di Marzio, Macromolecules, 24, 1595 (1991).

15. J. F. Tassin, L. Monnerie, L. J. Fetters, Polym. Bull, 15, 165 (1986); K. Osaki, E. Takatori, M. Kurata, H. Ohnuma, T. Kotaka, Polym. J., 12, 947 (1986); A. Lee, R. P. Wool, Macromolecules, 20, 1924 (1987); J. F. Tassin, L. Monnerie, L. J. Fetters, Macromolecules, 21, 2404 (1988). 